\title{
DESCENTRALIZAÇÃO DA PRODUÇÃO DO CUIDADO PARA A RESOLUBILIDADE DAS DEMANDAS DAS PESSOAS COM TUBERCULOSE: 0 significado para o enfermeiro da estratégia de saúde da família.
}

\section{Thalyne Souza Freitas Pinheiro'; Me. Pricila Oliveira de Araújo²; Dra Maria Yaná Guimarães Silva Freitas ${ }^{3}$; Brenda Fadigas Carvalho ${ }^{4}$}

\author{
1. Bolsista PIBIC/FAPESB, Graduando em Enfermagem, Universidade Estadual de Feira de Santana, e-mail: \\ thalyne.sfpinheiro@gmail.com \\ 2. Orientador, Departamento de Saúde, Universidade Estadual de Feira de Santana, e-mail: poaraujos@ gmail.com \\ 3. Participante do Núcleo de Pesquisa Integrada em Saúde Coletiva (NUPISC), Departamento de Saúde, Universidade \\ Estadual de Feira de Santana, e-mail: yana.guimaraess@ gmail.com \\ 4. Participante do Núcleo de Pesquisa Integrada em Saúde Coletiva (NUPISC), Departamento de Saúde, Universidade \\ Estadual de Feira de Santana, e-mail: brendafadigas@gmail.com
}

PALAVRAS-CHAVE: Tuberculose; Produção do Cuidado; Descentralização.

\section{INTRODUÇÃO}

O controle da tuberculose (TB) ainda é um dos maiores desafios às políticas de saúde pública no Brasil e no mundo. Caracterizada como emergência global, diante da sua complexidade epidemiológica, a TB constitui a agenda de políticas governamentais de diversos países. No Brasil, o Ministério da Saúde, para alcançar as metas de redução dos índices deste agravo, estabeleceu o Programa Nacional de Controle da Tuberculose (PNCT), em 2006. Articulado com a Política Nacional de Atenção Básica (PNAB), o PNCT procura desde então, descentralizar as ações de diagnóstico e tratamento da TB para a atenção básica (COELHO et al., 2014).

No caso das pessoas com TB, a produção do cuidado compreende não somente o uso dos anti-tuberculostáticos em si, mas a observância dos outros aspectos não medicamentos e que influenciam sobremaneira para a cura da doença e a proteção contra novas reinfecções tais como alimentação, sono e repouso, alcoolismo, etc. Mesmo tendo um tratamento medicamentoso eficaz, a tuberculose ainda apresenta altos índices de incidência, prevalência e mortalidade que podem estar relacionados a fatores organizacionais do sistema de saúde e às próprias condições de vida precária das pessoas, evidenciando assim uma fragilidade na resolubilidade do agravo (ARCÊNIO et al., 2009).

Diante da complexidade epidemiológica, da relevância da tuberculose e dos obstáculos para a produção do cuidado das pessoas com TB dentro dos serviços de saúde, observa-se a importância de discutir a descentralização da produção do cuidado, com vistas à resolubilidade das demandas das pessoas com TB, ressaltando que em Feira de Santana o PCT é implementado em um centro de referência especializado, o que compromete a resolubilidade do acompanhamento do usuário em tratamento da TB. Como o PCT deve ser operacionalizado na Atenção Básica, estratégias no referido município estão sendo implementadas para viabilizar a descentralização das ações e, neste sentido, faz-se necessário conhecer o que os enfermeiros da ESF pensam sobre tal processo de descentralização, emergindo o seguinte questionamento: qual o significado da descentralização da produção do cuidado para a resolubilidade das demandas das pessoas com TB na ótica do enfermeiro da ESF? Objetivando, Compreender o significado da descentralização da produção do cuidado para a resolubilidade das demandas das pessoas com TB, na ótica do enfermeiro da Estratégia de Saúde da Família.

\section{METODOLOGIA}


Estudo de abordagem qualitativa, do tipo exploratório descritivo. O estudo foi realizado no município de Feira de Santana - BA, em cinco Unidades de Saúde da Família. Participaram da pesquisa, cinco enfermeiros, com no mínimo seis meses de atuação na USF e que participaram da capacitação para a descentralização do Programa de Controle da Tuberculose no munícipio, realizada pela Secretaria Municipal de Saúde. Os dados foram coletados através de entrevista semiestruturada e analisados pelo método de análise de conteúdo de Bardin, que é dividida em três etapas (BARDIN, 2010), a pré-análise ocorreu a organização dos dados, as entrevistas gravadas foram transcritas, para obter as primeiras impressões do conteúdo. A análise ou exploração do material, realizou-se uma leitura mais aprofundada e sistemática dos dados, abstraindo os núcleos dos sentidos dos dados empíricos, emergindo, assim, duas categorias de análise: (1) "A descentralização é trabalhosa, mas acredito que vai dar certo, tem muitas vantagens para os pacientes"; (2) "A Descentralização só é ruim porque a gente não tem suporte". E por fim, a fase final, os dados obtidos foram articulados com a fundamentação teórica, levando em consideração a questão de pesquisa e o objetivo do estudo. Cumpriu-se a Resolução no 466 de 12 dezembro de 2012 (BRASIL, 2012).

\section{RESULTADOS E DISCUSSÃO}

Participaram desse estudo cinco enfermeiros, quatro do sexo feminino e um do sexo masculino, faixa etária com média de 33 anos e uma média de quatro anos de formação. Quanto ao vínculo empregatício, todos são cooperados e apenas dois não possuem pós graduação.

A partir dos dados coletados emergiram duas categorias de análise: (1) "A descentralização é trabalhosa, mas acredito que vai dar certo, tem muitas vantagens para os pacientes"; (2) "A Descentralização só é ruim porque a gente não tem suporte".

$\mathrm{Na}$ primeira categoria as falas dos entrevistados relatam que a descentralização tem muitos pontos positivos, principalmente para o benefício do paciente como: o acesso geográfico, o vínculo com a unidade e os profissionais, além de permitir um maior controle e vigilância a esse tratamento. Desta forma, a maioria dos enfermeiros relatam a relevância da iniciativa do município em iniciar o processo de descentralização da produção do cuidado à pessoa com TB.

Desde a integração do PNCT com a Política Nacional da Atenção Básica (PNAB), a busca pela intensificação da descentralização das ações do PNCT tem sido uma das prioridades dos municípios que ainda possuem essa assistência centralizada (ANDRADE et al., 2017), como é o caso do munícipio pesquisado, que está capacitando as equipes da ESF para que se torne uma realidade.

A preconização do PNCT em relação à horizontalização das ações do PCT, como controle, vigilância, prevenção e tratamento da doença para a ESF, vislumbra a relevância do cuidado a essas pessoas adoecidas (ANDRADE et al.,2017). O usuário do serviço terá como vantagens o acesso facilitado na USF, o vínculo com os profissionais, o que reafirma a responsabilidade da atenção primária no diagnóstico, acompanhamento e tratamento das pessoas com TB, além da avaliação dos contactantes.

A partir das falas dos enfermeiros que estão a frente dessas unidades, observamos a sensibilização dos mesmos em relação ao seu papel, mesmo que a descentralização represente mais um acúmulo de função para eles.

Nessa perspectiva, Mizuhira e outros (2015), afirmam que a Atenção Básica, mais especificamente a ESF, é a porta de entrada dos serviços de saúde e o elo com os demais níveis de atenção, como preconizado pelo MS, devido à forma como os mesmos são organizados, objetivando a vigilância e promoção da saúde. Desta forma, ratifica-se a responsabilidade da ESF no acompanhamento à pessoa com TB. 
A descentralização das ações do PNCT traz ao centro da discussão as dimensões da produção do cuidado em saúde, que discutem o acesso, o vínculo, o acolhimento, responsabilização e resolubilidade das demandas dos usuários

Nesse sentido, as falas dos entrevistados mostram que os enfermeiros de forma consciente, sinalizam que um dos pontos positivos da descentralização é justamente a interação do usuário e de suas redes de apoio com a USF. Assim, se configura o vínculo, usuários x enfermeiros $\mathrm{x}$ equipe de saúde, interligados através do agente comunitário, que facilita $\mathrm{o}$ processo de acompanhamento e tratamento as pessoas com TB, já que o serviço fica próximo a sua residência, além do que, na ESF, diferente de um centro especializado, elas têm um leque de programas que podem ser utilizados como, planejamento familiar, saúde da mulher ou do homem, dentre outros, e não apenas o tratamento da TB.

Em linhas gerais, observamos que a descentralização no município em estudo tem inúmeras potencialidade para acontecer de forma positiva, mas como qualquer situação, existem pontos desfavoráveis como: questões estruturais e de funcionamento do programa, armazenamento e transporte dos materiais coletados, que se configuram como desafios aos gestores e aos profissionais dos serviços de saúde para a prestação de uma atenção de qualidade, emergindo a seguinte categoria: "A Descentralização só é ruim, porque a gente não tem suporte".

Sendo assim, elegida como porta de entrada aos serviços de saúde, as USF são responsáveis pela prevenção, diagnóstico, tratamento e acompanhamento das pessoas com TB (BRASIL, 2009). Para isso, precisam estar devidamente preparadas, tanto em relação aos profissionais de saúde, que necessitam do aparato teórico para a atenção clínica, quanto relacional, como a escuta qualificada, acolhimento e vínculo de forma a garantir adesão das pessoas com TB.

Outro desafio que as unidades terão na implementação do PCT é dispor de transporte acessível para que haja o deslocamento dos exames coletados na unidade para o centro de referência (baciloscopia do escarro) para serem avaliados. Como as unidade não têm um carro próprio, isso se torna uma das preocupações dos entrevistados, inclusive porque o material, se não armazenado de forma correta, até o deslocamento para o CSE, poderá ter os resultados alterados.

A baciloscopia do escarro, quando realizada de forma correta, em todas as suas etapas, permite a detecção de $60 \%$ a $80 \%$ dos casos de tuberculose pulmonar, o que e importante epidemiologicamente, pois os casos bacilíferos são os responsáveis pela manutenção da cadeia de transmissão (BRASIL, 2009).

Conforme o Ministério da Saúde, (BRASIL, 2009) é de responsabilidade da unidade de saúde a coleta, conservação e transporte do escarro. Sendo assim, é necessário que seja seguida as orientações quanto a quantidade e qualidade da amostra. Para isso é preciso orientar a pessoa a cuspir após o escarro ser proveniente de tosse vigorosa. A armazenagem desse escarro, deve ser em coletores plásticos de boca larga, e a unidade de saúde deve mantê-los sob refrigeração até serem transportados para o laboratório lembrando que, durante o transporte, eles devem continuar sob refrigeração em isopor com gelo reciclável e protegidos da luz solar.

Ainda, para melhor atender mais um programa de saúde que, diferente dos outros, tratase de uma doença de alta transmissibilidade através do ar, é preciso que o fluxo de entrada e saída das pessoas nas USF seja otimizado, de forma a priorizar o atendimento dos sintomas respiratórios.

Desta maneira, para que haja descentralização das ações do PCT de forma resolutiva é preciso superar os desafios acima discutidos para o cumprimento das metas estabelecidas pelo município e pelo MS. 


\section{CONSIDERAÇÕES FINAIS}

A percepção dos enfermeiros sobre o processo de descentralização das ações do PCT para a ESF envolve um significado positivo para acompanhamento e tratamento das pessoas com tuberculose, já que a USF localiza-se no território de moradia do usuário, facilitando o acesso geográfico, pelo vínculo com os profissionais de saúde que favorece a adesão às terapêuticas, além da possibilidade de inserção em outros programas de saúde, para além do PCT.

Apesar dos entrevistados reconhecerem os aspectos positivos da descentralização, eles sinalizam para aspectos importantes que devem observados na ESF, principalmente, pelos gestores, de forma a garantir a qualidade do cuidado, bem como promover a resolubilidade das ações que tragam respostas às demandas das pessoas com tuberculose.

Assim, diante do exposto, os entrevistados acenam positivamente para a descentralização, ao passo que apontam desafios que devem ser superados de forma a garantir resolubilidade na produção do cuidado das pessoas com TB, necessitando, portanto, de estudos futuros para discutir como o PCT está sendo implementado na ESF no município estudado.

\section{REFERÊNCIAS}

ANDRADE H. S. et al. Avaliação do Programa de Controle da Tuberculose: um estudo de caso. Saúde Debate, Rio de janeiro, v. 41, n. Especial, p. 242-258, mar, 2017.

ARCÊNIO RA, et al. 2009. Barreiras econômicas na acessibilidade do doente ao tratamento da tuberculose em Ribeirão Preto (2007). In. Villa, TCS, Ruffino- Netto A. Tuberculose pesquisas operacionais, 1ed Ribeirão Preto, São Paulo: FUNPEC.

BARDIN, Laurence. Análise de conteúdo, Lisboa: Edições 70, 2010.

BRASIL, Ministério da Saúde. Hanseníase no Brasil: dados e indicadores epidemiológicos selecionados. Secretaria de Vigilância em Saúde. Departamento de Vigilância Epidemiológica, 2009.

BRASIL. Ministério da Saúde. Conselho Nacional de Saúde. Resolução No 466, de 12 de Dezembro de 2012.

COELHO AA, et al. Atenção à tuberculose: estudo de avaliabilidade. Rev. Latino-Am. Enfermagem, 2014.

FEIRA DE SANTANA, 2014. Secretária de Saúde. Plano Municipal de Saúde 2014 - 2017 Feira de Santana.

MIZUHIRA, Vanessa Fujino, et al. Procura da atenção para o diagnóstico da tuberculose. Arq. Ciênc. Saúde, 2015. 